\title{
The discovery of hard X-ray emission in the persistent flux of the Rapid Burster
}

\author{
M. Falanga ${ }^{1}$, R. Farinelli ${ }^{2}$, P. Goldoni ${ }^{1}$, F. Frontera ${ }^{2,3}$, A. Goldwurm $^{1}$, and L. Stella ${ }^{4}$ \\ ${ }^{1}$ CEA Saclay, DSM/DAPNIA/Service d'Astrophysique (CNRS FRE 2591), 91191, Gif-sur-Yvette, France \\ e-mail: mfalanga@cea.fr \\ 2 Dipartimento di Fisica, Università di Ferrara, via Paradiso 12, 44100 Ferrara, Italy \\ 3 IASF - CNR, Sezione di Bologna, via Gobetti 101, 40129 Bologna, Italy \\ 4 Osservatorio Astronomico di Roma, via Frascati 33, 00040 Monteporzio Catone, Italy
}

Received 6 May 2004 / Accepted 6 July 2004

\begin{abstract}
We report the first detection with INTEGRAL of persistent hard X-ray emission (20 to $100 \mathrm{keV}$ ) from the Rapid Burster (MXB 1730-335), and describe its full spectrum from 3 to $100 \mathrm{keV}$. The source was detected on February/March 2003 during one of its recurrent outbursts. The source was clearly detected with a high signal to noise ratio during the single pointings and is well distinguished from the neighboring source GX 354-0. The 3-100 keV X-ray spectrum of the persistent emission is well described by a two-component model consisting of a blackbody plus a power-law with photon index $\sim 2.4$. The estimated luminosity was $\sim 8.5 \times 10^{36} \mathrm{erg} \mathrm{s}^{-1}$ in the $3-20 \mathrm{keV}$ energy band and $\sim 1.3 \times 10^{36} \mathrm{erg} \mathrm{s}^{-1}$ in the $20-100 \mathrm{keV}$ energy range, for a distance of $8 \mathrm{kpc}$.
\end{abstract}

Key words. stars: individual: MXB 1730-335 - stars: neutron - X-ray: bursts - X-rays: general - X-rays: stars

\section{Introduction}

The Rapid Burster (MXB 1730-335, hereafter RB) is a low mass X-ray binary system (LMXB) located in the globular cluster Liller 1 at a distance of $\sim 8 \mathrm{kpc}$ (Ortolani et al. 1996) in the Galactic plane (Lewin et al. 1995, for a review). Unique in the Galaxy, the RB is the only LMXB known to produce both types I and II X-ray bursts. Type I bursts are characteristic of LMXBs hosting a low magnetic field neutron star (NS) and are known to be the result of explosive thermonuclear burning of accreted material on the surface of the NS. Type II bursts likely arise from the release of gravitational potential energy during sudden brief periods of higher accretion rate believed to be caused by a recurring accretion instability (e.g., Lewin et al. 1995). The RB is a recurring transient with outbursts which last for a few weeks followed by quiescent intervals. These intervals lasted for $\sim 200$ days until 1999, but have shrinked to $\sim 100$ days since 2000 (Masetti 2002).

One of the major problems in studying the persistent emission from this source is its proximity $\left(0.5^{\circ}\right.$ apart $)$ to the bright and variable LMXB 4U 1728-34 (GX354-0). As a consequence, it has never been possible to determine the persistent emission from the RB in the hard X-ray energy range, due to the low angular resolution of the collimator detectors used so far. Among the most recent results on the RB, we wish to mention those obtained with the Proportional Counter Array (PCA) aboard the Rossi X-ray Timing Explorer (RXTE) satellite (Guerriero et al. 1999), and those obtained with the BeppoSAX satellite (Masetti et al. 2000). Guerriero et al. (1999) monitored four outbursts of the source occurred between 1996 and 1998, detecting type I bursts, type II bursts, and the source persistent emission up to $20 \mathrm{keV}$. The persistent emission level was estimated offsetting the satellite pointing by $0.5^{\circ}$ away from GX354-0. They found that the $2.5-20 \mathrm{keV}$ persistent emission spectrum was well described by a multicomponent model consisting of two blackbodies (вв), plus a power-law (PL), noticing that up to $10 \mathrm{keV}$ the PL is not needed. Masetti et al. (2000) mainly studied in the broad $0.1-200 \mathrm{keV}$ energy band the evolution of the spectral properties of the bursting emission. The persistent emission spectrum was investigated in the range from 1 to $10 \mathrm{keV}$, also finding that a 2 вв model was suitable to describe the data. At energies higher than $10 \mathrm{keV}$, a residual contamination from GX 354-0 prevented an unbiased estimate the persistent emission flux level and spectrum from being performed with the Phoswich Detection System (PDS). Mahasena et al. (2003) observed type II bursts from th RB with ASCA in 1998 and 1999, and found that both the burst and persistent emission spectra, in the $1-10 \mathrm{keV}$ energy range, could be fitted with a two-component model consisting of a multi-color disc model and a blackbody.

We observed the RB in hard X-rays, using both the imager IBIS Ubertini et al. 2003 and the module 2 of the X-ray monitor JEM-X (Lund et al. 2003) on board the INTEGRAL satellite (Winkler et al. 2003). Thanks to their high angular 


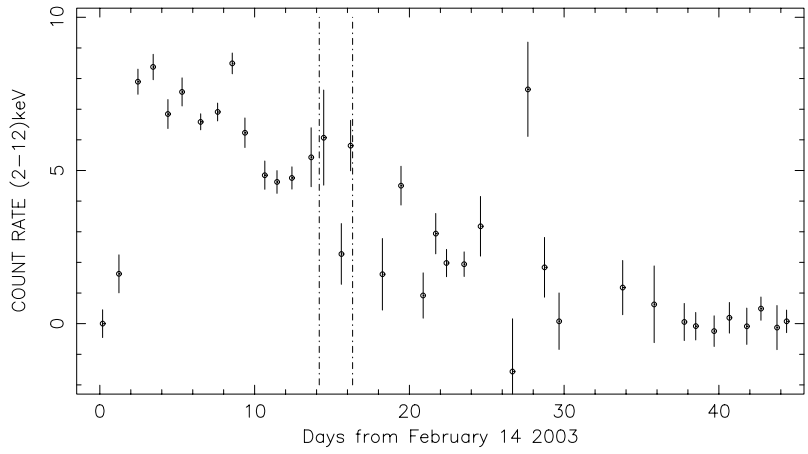

Fig. 1. RXTE/ASM light curve in the $2-12 \mathrm{keV}$ energy band (data averaged over 1-day intervals) of the February/March outburst of the RB. The vertical dashed lines indicate the times of the INTEGRAL observations.

resolution it was possible to separate the RB from GX 3540 . This represents a significant improvement over the previous measurements made with low spacial resolution instruments. Thus we were able to clearly measure the hard X-ray emission from the RB as distinct from that of the nearby GX 354-0.

We report here the first high energy detection of the RB during the decay phase from outburst to quiescence. The observation was obtained during the February/March 2003 outburst. The results are based on the $20-100 \mathrm{keV}$ data from IBIS, and on 3-20 keV data from JEM-X. In Sect. 2 we describe the observation, in Sect. 3 we present the spectral results, and in Sect. 4 we give the conclusion.

\section{INTEGRAL observation and data analysis}

The present dataset was obtained during a Target of Opportunity (ToO) observation of the Galactic center region, performed from February 28 to March 1, 2003 (satellite revolution 46), during the outburst decay phase of the RB. The observation spanned just over 2 days and caught the object as the flux level was decaying from an X-ray intensity corresponding to about $50 \%$ of the outburst peak down to quiescence. Figure 1 shows the 2-12 keV RB light curve obtained with the All-Sky Monitor (ASM) on board RXTE. Also shown in Fig. 1 are the time intervals in which the INTEGRAL observation was performed.

The IBIS imager is a coded mask instrument with an angular resolution of $12^{\prime} \mathrm{FWHM}$, a fully coded field of view (FOV) of $9^{\circ} \times 9^{\circ}$, and a partially coded FOV of $29^{\circ} \times 29^{\circ}$ at zero sensitivity. The detection plane consists of two detection layers, ISGRI and PICsIT. The upper detector, ISGRI (Lebrun et al. 2003), has a passband between $15 \mathrm{keV}$ and $1 \mathrm{MeV}$, but its best sensitivity is achieved between $20 \mathrm{keV}$ and $200 \mathrm{keV}$. The bottom detector, PICsIT (Di Cocco et al. 2003), has a passband between $\sim 200 \mathrm{keV}$ and $\sim 8 \mathrm{MeV}$. The JEM-X monitor is also a coded mask telescope with angular resolution of $3^{\prime}$ FWHM, a FOV of $4.8^{\circ}$ in diameter, and a partially coded FOV of $13.2^{\circ}$ in diameter. It covers the energy range from 3 to $25 \mathrm{keV}$.

Our observation consisted of 76 stable pointings (Science Windows, $\mathrm{ScW}$ ) of $\sim 2.2 \mathrm{ks}$ exposure each, with a $5 \times 5$ dithering pattern. Due to the dithering, which implied a change of the pointing direction, the RB was not always in the JEM-X field

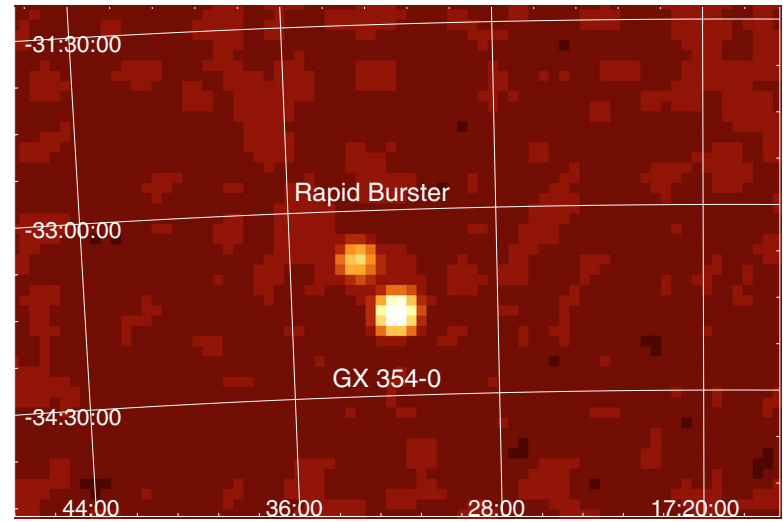

Fig. 2. The $20-40 \mathrm{keV}$ IBIS/ISGRI mosaicked, deconvolved sky image of the $\sim 173 \mathrm{ks}$ observation. Image size is $\sim 6^{\circ} \times 4^{\circ}$, centered at the RB position. The pixel size is $5^{\prime}$. The RB and GX 354-0 were detected at a significance of $\sim 37.7 \sigma$ and at $\sim 96 \sigma$, respectively.

of view. The RB position offsets, which ranged from $1^{\circ}$ to $9^{\circ}$, were used in the data extraction. For IBIS, the total exposure time was $\sim 173 \mathrm{ks}$. For JEM-X, for which the data extraction was restricted to pointings with an offset $\lesssim 3^{\circ}$, the total exposure time was $\sim 46 \mathrm{ks}$. Due to the high angular resolution of IBIS and JEM-X the RB was well separated from GX 354-0. The spectrometer (SPI) was not used since its $2^{\circ}$ angular resolution is inadequate in this case.

The data reduction was performed using the standard Offline Science Analysis (OSA) version 3.0 distributed by the INTEGRAL Science Data Center (Courvoisier et al. 2003). The algorithms used in the analysis are described by Goldwurm et al. (2003). For all instruments, the newest available (OSA 3.0) response matrices were used. For each ScW we extracted 16 channel spectra for IBIS and 256 channel spectra for JEM-X. Single pointings were deconvolved and analyzed separately, and then mosaicked. For each $\mathrm{ScW}$, source positions are determined by fitting a 2D Gaussian function to each peak in the deconvolved image. Source spectra are then obtained by a simultaneous fit of all sources detected in the image together with the background. The spectrum averaged on the total exposure time was obtained from those of the ScWs by weighting them for the exposure time of the individual pointings. The spectra were analyzed with the XSPEC v. 11.2.0 software package (Arnaud 1996).

\section{Results}

\subsection{Image and light curve properties of the $R B$}

IBIS

Figure 2 shows a significance map of the sources detected in the region around the RB position in the $20-40 \mathrm{keV}$ energy range. Two sources are clearly detected at a significance level of $\sim 37.7 \sigma$ for the RB and $\sim 96 \sigma$ for GX 354-0, respectively. At higher energies, $40-80 \mathrm{keV}$, the confidence level was $\sim 7 \sigma$ for the RB and $\sim 50 \sigma$ for GX 354-0. With the imaging procedure, from the IBIS data the derived celestial position of the RB is given by $(\mathrm{J} 2000) \mathrm{RA}=17^{\mathrm{h}} 33^{\mathrm{m}} 23^{\mathrm{s}} .81$, Dec $=-33^{\circ} 23^{\mathrm{m}} 36^{\mathrm{s}} .4$, 


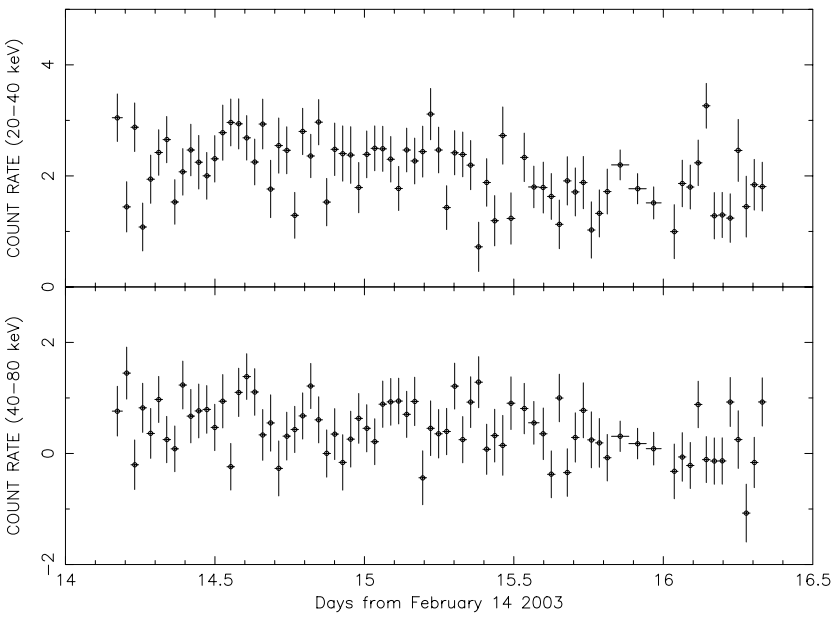

Fig. 3. IBIS/ISGRI light curve of the RB in the $20-40 \mathrm{keV}$ (top panel) and $40-80 \mathrm{keV}$ (bottom panel) energy bands. Each data point is averaged over one $\mathrm{ScW}$, and the count rate was extracted directly from the source reconstructed $\mathrm{ScW}$ image. Times are expressed in days starting from the outburst onset on February 14, 2003.

while that of GX $354-0$ is given by RA $=17^{\mathrm{h}} 31^{\mathrm{m}} 57^{\mathrm{s}} .72$, Dec $=-33^{\circ} 50^{\mathrm{m}} 5^{\mathrm{s}} .6$. The source position offset with respect to the catalog position is $\sim 0.34^{\prime}$ for the $\mathrm{RB}$, and $0.07^{\prime}$ for GX 354-0. This is within the $90 \%$ confidence level assuming the source location error given by Gros et al. (2003). The derived angular distance between the two sources is $\sim 32^{\prime}$.

The light curve of the RB integrated on each pointing in the same energy bands as above is shown in Fig. 3. The mean count rate in the $20-40 \mathrm{keV}$ and $40-80 \mathrm{keV}$ energy bands is $\sim 2.3 \mathrm{cts} / \mathrm{s}$ and $\sim 0.5 \mathrm{cts} / \mathrm{s}$, respectively. Above $100 \mathrm{keV}$ the $\mathrm{RB}$ was not detected at a statistically significant level either in single exposure or in the total exposure time. The IBIS spectrum and background are fitted simultaneously and extracted for each pointing and energy band. We excluded all channels below $20 \mathrm{keV}$ and above $100 \mathrm{keV}$.

\section{JEM-X}

At offset angles $\lesssim 3^{\circ}$ the RB was detected in each $\mathrm{ScW}$ at significance levels of $\sim 15-37 \sigma$, depending on the angular distance from the spacecraft pointing direction. Thanks of the hight angular resolution $\left(3^{\prime}\right)$ of $\mathrm{JEM}-\mathrm{X}$, the $\mathrm{RB}$ is not contaminated from GX 354-0. The derived light curve, in addition to the persistent emission, shows 11 bursts of duration ranging from $10 \mathrm{~s}$ to $60 \mathrm{~s}$. The $3-18 \mathrm{keV}$ count rates at the burst peak range from $\sim 29 \mathrm{cts} / \mathrm{s}$ to $89 \mathrm{cts} / \mathrm{s}$ (see the strongest burst in Fig. 4).

The statistics of the data however did not allow us to use the hardness ratio to determine whether the bursts are of type I or II. To get a reasonable signal to noise ratio, the burst light curves were binned at $5 \mathrm{~s}$, and this prevented us from looking at possible "ringing" effects in the burst timing profile, which are characteristic of type II bursts (see, e.g., Masetti et al. 2000). Because of multiple data gaps in the JEM-X light curve of the RB, we could not establish whether the expected linear relation between the X-ray energy in a burst and the time interval to the following burst (" $E-\Delta t$ " relation, Lewin et al. 1976)

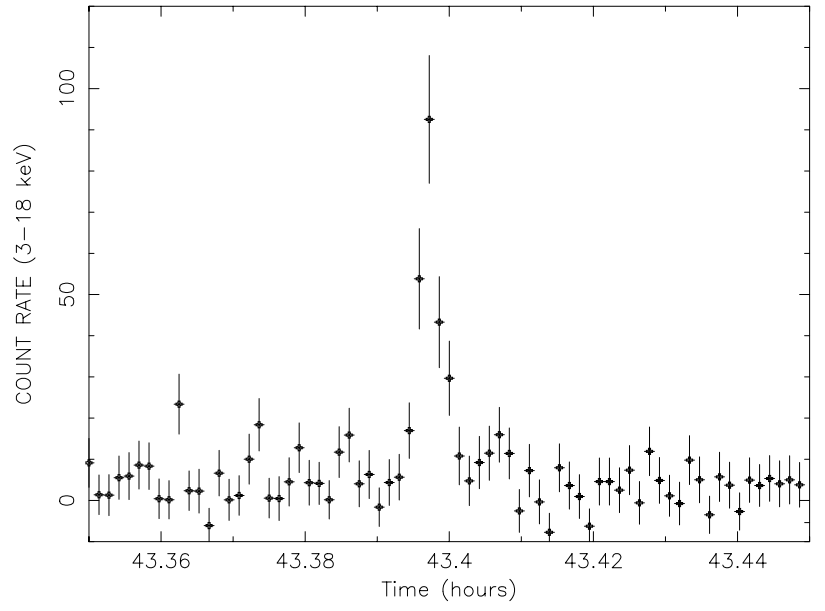

Fig. 4. JEM-X 3-18 keV count rate light curve of the strongest burst. Time is expressed in hours from the start of the observation. The bin time is $5 \mathrm{~s}$.

is satisfied by our burst data. For the same reason, we cannot easily classify the detected bursts according to the results by Guerriero et al. (1999), which predict type I bursts at particular epochs from the outburst onset. On the basis of the luminosity of the persistent emission $\left(<2 \times 10^{37} \mathrm{erg} \mathrm{s}^{-1}\right)$ in correspondence of the observed bursts, according to Guerriero et al. (1999), type II bursts are favored. However, the estimated the 3-18 $\mathrm{keV} \alpha$ ratio between the persistent and bursting fluence emission (46 \pm 2$)$, which is well above the minimum value found in the case of type I X-ray bursts (Lewin et al. 1995), would point to a type I burst origin of the observed events.

\subsection{Persistent emission and $X$-ray burst spectra}

The mean 3-100 keV spectrum of the Rapid Burster persistent emission was obtained by using all the useful JEM-X and IBIS data except those corresponding to the 11 time intervals in which the X-ray bursts were observed. A constant factor was also included in the fit to take into account both the uncertainty in the instruments cross-calibration and the fact that IBIS and JEM-X have different exposure times: the last point in fact can introduce systematic effects in the fitting result, expecially if the source is characterized by a bursting behaviour at the time of the observations. We fixed this constant equal to 1 for JEM-X, allowing it to vary for IBIS. We found that the spectrum is well described by a photo-electrically absorbed multi-component model consisting of a BB plus a PL plus a Gaussian emission line centered at $6.5 \mathrm{keV}$. We were not able to constrain the interstellar column density $N_{\mathrm{H}}$, so $N_{\mathrm{H}}$ was frozen at $1.6 \times 10^{22} \mathrm{~cm}^{-2}$ in our fit, the Galactic value reported in the radio maps of Dickey \& Lockman (1990) along the source direction. This value is consistent with that measured by Masetti et al. (2000). The significance of the emission line is very high, as also testified by the $\chi^{2} /$ (degrees of freedom, d.o.f. $)=178 / 138$ in the case the line is not included in the fit to $\chi^{2} /$ d.o.f. $=115 / 135$ after its inclusion, with a probability of chance improvement of $\sim 10^{-14}$. The best fit parameters of the model together with the $90 \%$ confidence single 
Table 1. Spectral fit with blackbody, Gaussian \& power law components.

\begin{tabular}{lc}
\hline \hline Parameter & Value \\
\hline$N_{\mathrm{H}}^{a}$ & $1.6 \times 10^{22} \mathrm{~cm}^{-2}$ \\
Blackbody $T_{\mathrm{bb}}$ & $2.2_{-0.1}^{+0.1}(\mathrm{keV})$ \\
Blackbody $R_{\mathrm{bb}}^{b}$ & $1.4_{-0.1}^{+0.1}(\mathrm{~km})$ \\
Gaussian $E_{l}$ & $6.5_{-0.2}^{+0.2}(\mathrm{keV})$ \\
Sigma $\sigma_{l}$ & $0.7_{-0.3}^{+0.3}(\mathrm{keV})$ \\
Equivalent width & $470_{-150}^{+200}(\mathrm{eV})$ \\
Photon index $\Gamma$ & $2.4_{-0.1}^{+0.1}$ \\
$\chi_{\text {red }}^{2}$ & $0.85(135 \mathrm{~d} . \mathrm{o.f})$. \\
$L_{x}^{b}(3-100 \mathrm{keV})$ & $9.8 \times 10^{36} \mathrm{erg} \mathrm{s}{ }^{-1}$ \\
$L_{\mathrm{PL}} / L_{\text {tot }}(3-100 \mathrm{keV})$ & 0.45 \\
\hline${ }^{a}$ Fixed for the fit. & \\
${ }^{b}$ Assuming a distance of $8 \mathrm{kpc}$. & \\
\end{tabular}

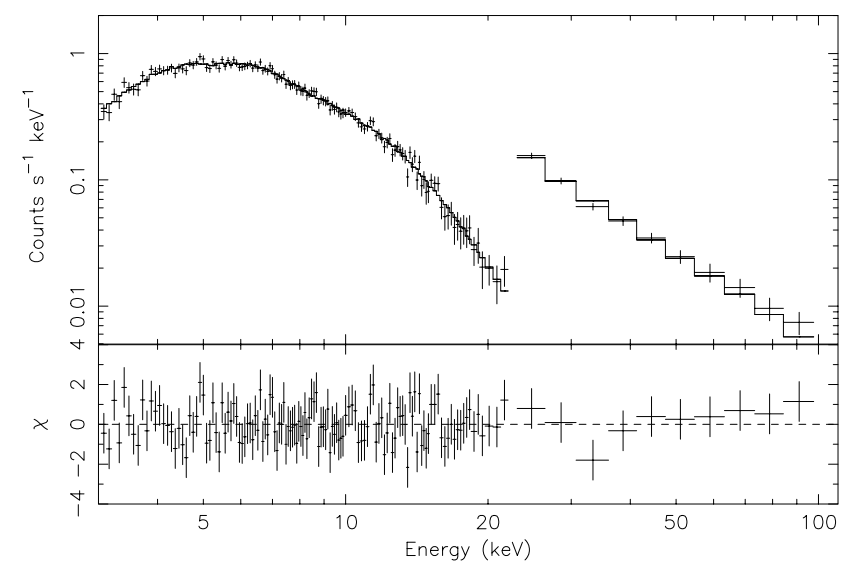

Fig. 5. The 3-100 keV count rate spectrum of the RB with the best fit model вB + GAussian + PL. In the bottom panel are shown the residuals between the data and the model in units of sigma.

parameter errors are reported in Table 1, while the count rate and the $E F(E)$ spectrum along with the single fit components and the residuals on the count spectrum to the best fit model are shown in Figs. 5 and 6.

Assuming that the PL is due to Comptonization of soft photons off high energy electrons, although we do not find any evidence for a high-energy cut-off at $100 \mathrm{keV}$, we attempted to put a lower limit on the temperature of the electron responsible for hard X-ray emission. For that, we substituted the simple PL, with the Comptonization model comprs of Poutanen \& Svensson (1996), assuming a spherical geometry and a multicolour disk blackbody (Mitsuda et al. 1984) seed photon spectrum. This model also provides a very good description of the data $\left(\chi^{2} /\right.$ d.o.f. $\left.=114 / 133\right)$ and gives a $2 \sigma$ lower limit of $90 \mathrm{keV}$ for the electron temperature, an opacity $\tau \sim 0.8$ and a inner edge disk temperature of $\sim 1.3 \mathrm{keV}$. Using the JEM-X data, we have also analyzed the X-ray burst spectrum. Because of the low statistics available for the single burst spectra, we summed them together deriving a mean spectrum. We found that a photo-electrically absorbed вв describes the data well $\left(\chi^{2} /\right.$ d.o.f. $\left.=65 / 57\right)$, with a temperature $k T_{\mathrm{bb}}=2.1 \pm 0.1$, which

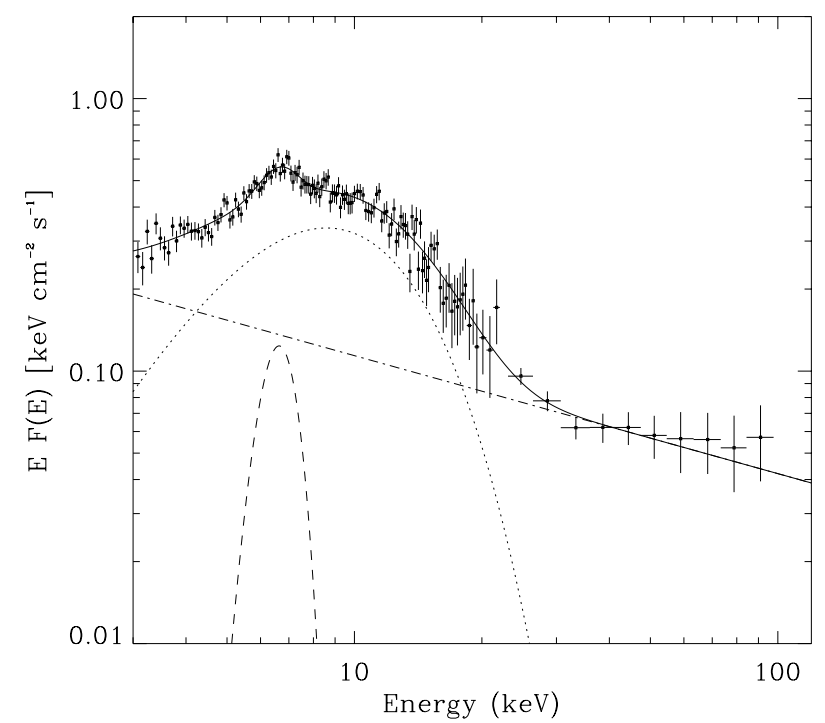

Fig. 6. Unabsorbed 3-100 $\mathrm{keV} \mathrm{EF}(\mathrm{E})$ spectrum of the RB along with the best fit model вв + Gaussian + PL. Different line styles show the single model components. Dotted: вв. Dotted-dashed: PL. Long-dashed: Gaussian.

is consistent with that estimated for the persistent emission (see Table 1), and a corresponding вв radius of $R_{\mathrm{bb}}=3.8_{-0.3}^{+0.3} \mathrm{~km}$. From the Bв model, we estimated an average burst luminosity of $\sim 4 \times 10^{37} \mathrm{erg} \mathrm{s}^{-1}$. However, the strongest burst reached a bolometric luminosity of $\sim 2 \times 10^{38} \mathrm{erg} \mathrm{s}^{-1}$.

To compare our spectral results with those obtained by Masetti et al. (2000), we also considered the X-ray burst spectrum with the persistent $\mathrm{X}$-ray emission subtracted. In this case, no significant change was observed in the вв temperature, and BB radius, while the mean burst bolometric luminosity is a little bit lower $\left(\sim 3 \times 10^{37} \mathrm{erg} \mathrm{s}^{-1}\right)$.

\section{Discussion and conclusion}

INTEGRAL has provided the first measurement of the persistent high energy X-ray emission from the RB without contamination from the source GX 354-0 located at $\sim 30^{\prime}$ from the RB. We observed X-ray emission from $3 \mathrm{keV}$ up to $100 \mathrm{keV}$ in the time period from 14 to 16 days from the outburst onset. The broad band persistent emission spectrum measured during the INTEGRAL ToO observation, is well described by the sum of a вв a Gaussian plus a PL model. The inferred вв temperature is $\sim 2.2 \mathrm{keV}$, while the photon index of the high energy PL component is $\sim 2.4$. The persistent emission source luminosity is $\sim 10^{37} \mathrm{erg} \mathrm{s}^{-1}$ in the energy range from $3-100 \mathrm{keV}$. No evidence for a cutoff in the power law component was found up to energies of $\sim 100 \mathrm{keV}$. Assuming for the hard spectral component a thermal Comptonization model, we get a lower limit for the electron temperature of $\sim 90 \mathrm{keV}$. However we cannot exclude that the hard X-ray spectral component is due to nonthermal Comptonization processes occurring in the source (see, e.g., Coppi 1999, for a review).

An interesting feature of the RB $3-100 \mathrm{keV}$ spectrum is that, when modeled by a BB plus PL, it is very similar to that of atoll sources in the hard state. Indeed, in some of these 
sources, during their hard states, the X-ray spectrum extends up to several hundreds of $\mathrm{keV}$ without any evidence of cutoff (e.g., KS 1731-260 and GX 354-0, Barret \& Vedrenne 1994; Aql X-1, Harmon et al. 1996). This behaviour at high energies is also similar to that of Black Hole Candidates in the so-called power-law gamma-ray state (Grove et al. 1998). The similarity of the observed RB spectrum to that of atoll sources in hard state would be consistent with our classification, discussed in Sect. 3.1 of the observed X-ray bursts as type I bursts. However in the atoll sources in their hard state the luminosity of the вв component is less than about $20 \%$ with respect to their total luminosity, while in our case the вв contributes to more than $50 \%$ of the total luminosity. A strong contribution of the Bв component to the total X-ray luminosity is instead typical of Z-sources (see, e.g., Di Salvo et al. 2000). This "anomaly" of the RB spectrum with respect to that of the other atoll sources could however be related to the particular, and still not well understood, physical environment of the source (perhaps the "propellereffect" caused by the magnetic field of the neutron star). Indeed we do not know why the RB, and only the RB, displays both types I and II X-ray bursts in continously changing times and modality. Thus the fact that the RB cannot be fully classified as a "classical" atoll source is not surprising, given the peculiarity of the RB. However, with INTEGRAL, a new step towards understanding the source has been taken. A step forward could be made by extending the spectral study at energies well above $100 \mathrm{keV}$, in order to search for any cutoff or to test whether nonthermal Comptonization processes (well observed and studied in Black Hole Candidates) also occur in this unique source. This can be achieved with dedicated INTEGRAL observations of another, possibly stronger, outburst of the Rapid Burster.

Acknowledgements. M.F. is grateful to E. W. Bonning for valuable suggestions and assistance with the manuscript, and acknowledges financial support from the France Spatial Agency (CNES).

\section{References}

Arnaud, K. A. 1996, in Astronomical Data Analysis Software and Systems V, ed. G. H. Jacoby, \& J. Barnes (San Francisco: ASP), ASP Conf. Ser., 101, 17

Barret, D., \& Vedrenne, G. 1994, ApJS, 92, 505

Courvoisier, T. J.-L., Walter, R., Beckmann, V., et al. 2003, A\&A, 411, L57

Coppi, P. S. 1999, in High Energy Processes in Accreting Black Holes, ed. J. Poutanen, \& R. Svensson (San Francisco: ASP), ASP Conf. Ser., 161,375

Di Cocco, G., Caroli, E., Celesti, E., et al. 2003, A\&A, 411, L189

Di Salvo, T., Stella, L., Robba, R., et al. 2000, ApJ, 544, L119

Dickey, J. M., \& Lockman, F. J. 1990, ARA\&A, 28, 215

Goldwurm, A., David, P., Foschini, L., et al. 2003, A\&A, 411, L223

Gros, A., Goldwurm, A., Cadolle-Bel, M., et al. 2003, A\&A, 411, L179

Grove, J. E., Johnson, W. N., Kroeger, R. A., et al. 1998, ApJ, 500, 899

Guerriero, R., Fox Kommers, J., et al. 1999, MNRAS, 307, 179

Harmon, B. A., Wilson, C. A., Tavani, M., et al. 1996, A\&AS, 120, 197

Lebrun, F., Laray, J.-P., Lavocat, Ph., et al. 2003, A\&A, 411, L141

Lewin, W. H. G., Doty, J., Clark, G. W., et al. 1976, ApJ, 207, L99

Lewin, W. H. G., van Paradijs, J., \& Taam, R. E. 1995, in X-ray Binaries, ed. W. H. G. Lewin, J. van Paradijs, \& E. P. J. van den Heuvel (Cambridge Univ. Press), 175

Lund, N., Budtz-Jørgensen, C., Westgaard, N. J., et al. 2003, A\&A, 411, L231

Mahasena, P., Inoue, H., Asai, K., \& Dotani, T. 2003, PASJ, 55, 827

Masetti, N. 2002, A\&A, 381, L45

Masetti, N., Frontera, F., Stella, L., et al. 2000, A\&A, 306, 134

Mitsuda, K. K., Inoue, H., Koyama, K., et al. 1984, PASJ, 36, 741

Ortolani, S., Bica, E., \& Barbuy, B. 1996, A\&A, 306, 134

Poutanen, J., \& Svensson, R. 1996, ApJ, 470, 249

Ubertini, P., Lebrun, F., Di Cocco, G., et al. 2003, A\&A, 411, L131

Winkler, C., Courvoisier, T. J.-L., Di Cocco, G., et al. 2003, A\&A, 411, L1 